\title{
Candidate fossil groups in the CFHTLS: a probabilistic approach ${ }^{\star}$
}

\author{
C. Adami ${ }^{1}$, F. Sarron ${ }^{2}$, N. Martinet ${ }^{1}$, and F. Durret ${ }^{3}$ \\ 1 Aix Marseille Univ, CNRS, CNES, LAM, Marseille, France \\ e-mail: christophe .adami@lam.fr \\ 2 School of Physics and Astronomy, University of Nottingham, Nottingham NG7 2RD, UK \\ 3 Sorbonne Université, CNRS, UMR 7095, Institut d'Astrophysique de Paris, 98bis Bd Arago, 75014 Paris, France
}

Received 10 July 2019 / Accepted 19 April 2020

\begin{abstract}
Context. Fossil groups (FGs) have been discovered 25 years ago, and are now defined as galaxy groups with an X-ray luminosity higher than $10^{42} h_{50}^{-2} \mathrm{erg} \mathrm{s}^{-1}$ and a brightest group galaxy brighter than the other group members by at least two magnitudes. However, the scenario of their formation remains controversial.

Aims. We propose here a probabilistic analysis of FGs, extracted from the large catalog of candidate groups and clusters previously detected in the CFHTLS survey based on photometric redshifts to investigate their position in the cosmic web and probe their environment.

Methods. Based on spectroscopic and photometric redshifts, we estimated the probability of galaxies to belong to a galaxy structure, and by imposing the condition that the brightest group galaxy is at least brighter than the others by two magnitudes, we computed the probability for a given galaxy structure to be a FG. We analyzed the mass distribution of these candidate FGs, and estimated their distance to the filaments and nodes of the cosmic web in which they are embedded.

Results. We find that structures with masses lower than $2.4 \times 10^{14} M_{\odot}$ have the highest probabilities of being fossil groups (PFG). Overall, structures with PFG $\geq 50 \%$ are located close to the cosmic web filaments (87\% are located closer than $1 \mathrm{Mpc}$ to their nearest filament). They are preferentially four times more distant from their nearest node than from their nearest filament.

Conclusions. We confirm that FGs have low masses and are rare. They seem to reside closely to cosmic filaments and do not survive in nodes. Being in a poor environment might therefore be the driver of FG formation because the number of nearby galaxies is not sufficient to compensate for the cannibalism of the central group galaxy.
\end{abstract}

Key words. galaxies: clusters: general - X-rays: galaxies: clusters - large-scale structure of Universe - galaxies: groups: general

\section{Introduction}

Fossil groups (FGs) are puzzling large-scale structures which present high X-ray luminosities but fewer bright optical galaxies than groups or clusters of galaxies. Ponman et al. (1994) reported the first observation of such an object. Jones et al. (2003) later defined FGs as extended X-ray sources with an X-ray luminosity of at least $L_{\mathrm{X}}=10^{42} h_{50}^{-2} \mathrm{erg} \mathrm{s}^{-1}$, and a brightest group galaxy (BGG) at least two magnitudes brighter than all other group members. An open question is the formation of these peculiar objects and why they present such a low amount of optically emitting matter.

An early explanation that has been proposed by Jones et al. (2003) is that FGs are the remnants of early mergers, and that they are cool-core structures which a long time ago accreted most of the large galaxies in their environment. Although this scenario was supported by some hydrodynamical simulations by D'Onghia et al. (2005), some clues also exist that FGs might be a temporary stage of group evolution before they capture more galaxies in their vicinity, as reported for instance by von Benda-Beckmann et al. (2008, see based on $N$-body simulations).

* Based on observations obtained with XMM-Newton, ESO Telescopes at the La Silla and Paranal Observatories, and MegaPrime/ MegaCam at the Canada-France-Hawaii Telescope (see acknowledgements for more details).
The situation is not simpler on the observational side, partly because we lack large samples of FGs, and partly because selection criteria differ. Fossil groups can be studied through their X-ray (e.g., Adami et al. 2018) or optical properties (e.g., Santos et al. 2007). Girardi et al. (2014) found identical behaviors for regular groups and FGs when they considered the relation between their X-ray and optical luminosities, which suggests that FGs contain the same amount of optical material than traditional groups, but that it is concentrated in a giant elliptical galaxy that has cannibalized most surrounding bright galaxies early on. La Barbera et al. (2009) also found that the optical properties of BGGs in FGs are identical to those of giant isolated field galaxies. Both analyses support the scenario that FGs are the result of a large dynamical activity at high redshift, but in an environment that is too poor for them to evolve into a cluster of galaxies through the hierarchical growth of structures. Based on Chandra X-ray observations, Bharadwaj et al. (2016) found that FGs are mostly cool-core systems, which adds to the other indications that these structures are now dynamically dead.

The most recent observations, however, tend to contradict these results. Kim et al. (2018) reported that NGC 1132 is a FG with an asymmetrical disturbed $\mathrm{X}$-ray profile, and suggested that it is not dynamically passive, as expected. Similarly, Lima Neto et al. (2020) discovered shells around the BGG of NGC 4104. Based on $N$-body simulations, they showed that this FG experienced a recent merger between its BGG and another bright galaxy with a mass of about $40 \%$ of that of the BGG. 
These two examples show exceptions to the FG nomenclature that might indicate more complex evolutionary scenarii.

One option to better understand the evolution of these systems is to perform a probabilistic analysis of FGs. We follow this approach here by making use of the large candidate cluster and group sample of Sarron et al. (2018). These authors identified a large number of structures in the $154 \mathrm{deg}^{2}$ of the Canada-FranceHawaii Telescope Legacy Survey (CFHTLS) by applying an adaptive Gaussian filtering in photometric redshift slices. This provided us with a large sample of groups from which it was possible to isolate FGs in a probabilistic way instead of studying particular objects, as is usually done in the case of FGs.

In the present analysis we also make use of the network of filaments of galaxies defined through topological criteria after the same Gaussian filtering as obtained by Sarron et al. (2019) to investigate the positions of the FGs in the cosmic web. This allows us to probe the direct vicinity of FG candidates in observational data for the first time.

The paper is structured as follows. In Sect. 2 we describe our data set. In Sect. 3 we derive the probabilities of the group candidates to be FGs. We measure the properties of the FGs, in particular their positions in the cosmic web in Sect. 4, and we conclude in Sect. 5.

Throughout the paper we adopt a $\Lambda \mathrm{CDM}$ cosmology with $\Omega_{\mathrm{m}}=0.30, \Omega_{\Lambda}=0.70$, and $\mathrm{H}_{0}=70 \mathrm{~km} \mathrm{~s}^{-1} \mathrm{Mpc}^{-1}$, and all cosmological distances are given in comoving Mpc.

\section{Data}

\subsection{CFHTLS}

Photometric redshifts are taken from the CFHTLS T0007 data release $^{1}$, which covers $154 \mathrm{deg}^{2}$ across four Wide fields (W1, W2, W3, and W4), observed in the $u^{*} g^{\prime} r^{\prime} i^{\prime} z^{\prime}$ filters with the MegaCam at the CFHT. The photo-zs are computed using the LePhare software (Arnouts et al. 1999; Ilbert et al. 2006) following the method presented in Coupon et al. (2009). LePhare computes photo-zs by fitting spectral energy distributions (SEDs) to the five-band magnitude measurements. In the present case, 62 SED galaxy templates were used. They were obtained through a linear interpolation between four templates from Coleman et al. (1980) and two starburst templates from Kinney et al. (1996) using the VIMOS-VLT Deep Survey (VVDS) spectroscopic sample (e.g., Le Fèvre et al. 2005). This interpolation allows us to accurately sample the color-redshift space.

Following the definition of Ilbert et al. (2006) and Coupon et al. (2009), the photo- $z$ dispersion was estimated using the normalized median absolute deviation (NMAD) estimator,

$\sigma_{\Delta z /\left(1+z_{\mathrm{s}}\right)}=1.48 \times \operatorname{median}\left(\frac{|\Delta z|}{\left(1+z_{\mathrm{s}}\right)}\right)$,

where $\Delta z=z_{\text {phot }}-z_{\mathrm{s}}$, where $z_{\text {phot }}$ and $z_{\mathrm{s}}$ correspond to the photometric and spectroscopic redshifts, respectively. We also define the catastrophic failure rate $\eta$ as the percentage of objects satisfying the criterion $|\Delta z| \geq 0.15 \times\left(1+z_{\mathrm{s}}\right)$.

For our analysis, we discarded any galaxy that falls into the mask of the CFHTLS T0007 data release. These masks correspond to areas with bright stars or artifacts, for which magnitude measurements are inaccurate. We thus avoid including objects with poor photo- $z$ quality that would degrade our sample.

\footnotetext{
1 https://www.cfht.hawaii.edu/Science/CFHTLS/T0007/
}

To further improve the redshift estimates of the CFHTLS Wide catalog, we correlated it with known spectroscopic redshifts from the Six-degree Field (6dF) galaxy survey (Jones et al. 2009), the VVDS (Le Fèvre et al. 2013), the VIMOS Public Extragalactic Redshift (VIPERS) Survey (Scodeggio et al. 2018), the VIMOS Ultra Deep (VUDS) Survey (Tasca et al. 2017), the Galaxy And Mass Assembly (GAMA) survey (Baldry et al. 2018), and the spectroscopic part of the Cosmic Evolution Survey (zCOSMOS; Lilly et al. 2009). These data were extracted through the ASPIC public database ${ }^{2}$. We additionally used the NED to complement this database and extracted all available spectroscopic redshifts in the $\mathrm{W} 1, \mathrm{~W} 2, \mathrm{~W} 3$, and W4 CFHTLS regions. We performed the cross-correlation in $\mathrm{RA}$, Dec coordinates within a 1 arcsec research box and retained the highest confidence spectroscopic redshift in case of multiple identifications. This led to the addition of $\sim 73000$ spectroscopic redshifts to the photometric catalogs. We also used these new spectroscopic redshifts to refine the quality check of the photo- $z$ catalog. Using Eq. (1), we estimated a statistical uncertainty of 0.07 after $3 \sigma$ clipping. We verified that the number of photo- $z$ catastrophic failures (computed before the $3 \sigma$ clipping) is small: only $1.6 \%$ of the galaxies present a difference between photometric and spectroscopic redshifts larger than our $3 \sigma$ clipping level. We also know that photometric redshift precision is sometimes degraded when cluster galaxies are considered (Guennou et al. 2010; Martinet et al. 2015), especially in massive clusters. Considering only galaxies within one Virial radius from the XXL-detected X-ray massive structures in the W1 field (Adami et al. 2018) and within the $z=[0.15 ; 0.70]$ range, we still have a precision of 0.07 . This does not affect our detection rate or purity too strongly, as was shown by Sarron et al. (2018).

Finally, we investigated the photometric redshift precision as a function of the parent cluster mass in order to verify that lowmass structures do not have particularly degraded photometric redshifts. This is not expected to be the case because the galaxy populations of these low-mass structures are more similar to the field populations than the ones of massive structures and are less affected by environmental effects. To do this, we also considered the previous XXL cluster sample and computed the photometric redshift precision as a function of the cluster X-ray temperature. Clusters colder than $4 \mathrm{keV}$ (relatively massive structures) exhibit a precision of $\sigma_{\Delta z /\left(1+z_{\mathrm{s}}\right)}=0.069$. This value decreases to 0.061 for clusters colder than $1 \mathrm{keV}$, that is, within the group regime. Photometric redshift precision therefore does not affect low-mass cluster detection more than high-mass cluster detection.

\subsection{CFHTLS AMASCFI group catalog}

The original group catalog was obtained by running the Adami, MAzure and Sarron Cluster FInder (AMASCFI) algorithm on the CFHTLS T0007 data. Details on the cluster and group candidate catalog and on the detection algorithm can be found in Sarron et al. (2018) and Adami \& Mazure (1999). Here, we report the salient points of the analysis and the few modifications we applied to the original catalog.

First, we cut the galaxy catalog into redshift slices of width typical of the photo- $z$ uncertainty and offset from each other by $\Delta z=0.05$. We then applied a kernel smoothing with an adaptive smoothing scale to each slice and identified the peaks in these two-dimensional density maps using Sextractor

\footnotetext{
2 http://cesam.lam.fr/aspic/
} 
(Bertin \& Arnouts 1996). A minimal spanning tree (MST, see, e.g., Adami \& Mazure 1999) was then applied to merge individual detections with a projected separation smaller than $1 \mathrm{Mpc}$ and a redshift difference smaller than $\Delta z=0.06$. Finally, we provided a mass estimate (Virial mass $M_{200}$ ) by considering a scaling relation between mass and richness, where the richness is defined as the number of passive galaxies brighter than $M^{*}+1.75$, where $M^{*}$ is the characteristic magnitude of the cluster. Here, passive galaxies are defined according to their best-fit template computed by LePhare when the redshift of the galaxy is fixed at the cluster redshift (see Sarron et al. 2018, for details). This scaling relation was obtained by cross-matching our detections with the X-ray catalogs of Gozaliasl et al. (2014) and Mirkazemi et al. (2015). We estimated the typical $M_{200}$ uncertainty to be $\sim 0.20-0.25$ dex in Sarron et al. (2018). Cluster redshifts were computed as the mean photometric redshift over individual detections linked by the MST, weighted by their mean galaxy densities (see Sarron et al. 2018, for details), and present an uncertainty of $\sigma_{z}=0.025 \times(1+z)$.

A mass-richness scaling relation might bias the mass of FGs toward lower values because they exhibit lower galaxy counts at the bright end of the galaxy luminosity function than regular groups (see, e.g., Zarattini et al. (2016)). We tested this hypothesis by computing the scaling relation between the richness of each structure and its total luminosity by summing the individual luminosities of cluster members with the same magnitude cut as for the richness definition $\left(\mathrm{M}^{*}+1.75\right)$. This richness-luminosity function follows a power law, and no statistical difference can be found between samples with different PFGs (higher than $15 \%$ and $50 \%$ and lower than $10 \%$ ), showing that our mass-richness relation is unlikely to bias the masses of the candidate FGs compared to other structures.

Sarron et al. (2018) computed the selection function for clusters with mass $M_{200}>10^{14} M_{\odot}$. The completeness and purity of this cluster candidate sample are overall about $80 \%$ and $90 \%$, respectively, for $z \leq 0.7$. However, these values are lower when very low mass structures are considered. Our best FG candidates (PFG $\geq 50 \%$ : 15 structures, see below) are mostly at $z \leq 0.4$ and have masses between $1.1 \times 10^{13}$ and $2.4 \times 10^{14} M_{\odot}$. Figure 1 of Sarron et al. (2018) rather suggests a completeness of $\leq 60 \%$ and a purity of $\leq 80 \%$ in this redshift-mass regime.

To compute the cluster probability membership of individual galaxies, we computed a photometric redshift probability distribution function (PDF hereafter) for each group in the AMASCFI catalog. This was made by summing the photometric redshift PDFs of individual galaxies that lie at a distance $d<0.5 \mathrm{Mpc}$ (at the group redshift) of the group center. The resulting distribution was then cut off at $z_{\text {inf }}=z_{\text {min,MST }}$ and $z_{\text {sup }}=z_{\text {max,MST }}$, the lower and upper redshifts of the individual two-dimensional detections linked by the MST. This was done to retain only the contribution of the group in the redshift PDF. We then computed the field contribution by summing the redshift PDFs of field galaxies and removed that contribution from our first estimates, thus forming the group photometric redshift PDF.

When multiple significant peaks separated by more than $0.05 \times(1+z)$ were found, we separated them, assigning the individual two-dimensional detections in the MST accordingly to each peak. We chose the most prominent peak to be that of the group and thus that defining its redshift PDF, while the other peaks were ignored. We also changed the group position and redshift accordingly, considering only the individual detections in the MST corresponding to the retained peak. We note that this refining process was marginal and only concerned a few percent of our original detections.

\subsection{Cosmic filament catalog}

When the spatial distribution of FGs was investigated relative to the cosmic web, we used the catalog of cosmic filaments and nodes detected by Sarron et al. (2019) in the CFHTLS. The skeleton (filament, nodes, and saddle points) reconstruction was performed as in Laigle et al. (2018), applying the DISPERSE algorithm of Sousbie (2011) to the two-dimensional galaxy distribution in photometric redshift slices. Details about the catalog we used can be found in Sarron et al. (2019). Briefly, given the uncertainty on the photo- $z$, slices were chosen to be $300 \mathrm{Mpc}$ thick. This is orders of magnitude larger that the typical radius of filaments $(\sim 1 \mathrm{Mpc})$, thus leading to projection effects in the slices that may result in false detections of filaments. An important part of the work by Sarron et al. (2019) therefore was to test the method performance.

We consider here what Sarron et al. (2019) called the "global reconstruction", where cosmic filaments and nodes are reconstructed in the entire field of view of each CFHTLS Wide field. Based on mock data, they showed that in the $0.15 \leq z<0.7$ redshift range this reconstruction is $\sim 70 \%$ complete and $\sim 90 \%$ pure. We refer to their work for details on the selection function computation.

In each two-dimensional slice, DISPERSE traces the filaments of the cosmic web as a set of segments joining what it identified in the discrete galaxy distribution as nodes (local maximum of the distribution) and saddle points. In the theory of structure formation, galaxy groups and clusters are expected to be found at the nodes of the cosmic web. In our case, considering the thickness of our two-dimensional slices, we note, however, that some apparent nodes might instead be due to the projection of filaments in two dimensions.

For each structure in the catalog of Sarron et al. (2018), we thus matched its position with that of the nodes of the twodimensional skeleton reconstructed at the best redshift of the structure. When a node fell within a circle of one Virial radius $\left(R_{200}\right)$ of the group, it was considered a match and the structure was considered as indeed being at a node of the cosmic web.

\section{Probabilistic approach for detecting fossil groups}

\subsection{Estimating the structure membership probability for a given galaxy}

In contrast to Adami et al. (2018), for example, where the cluster redshifts were known with a very high precision (spectroscopic redshifts), we here relied only on photometric redshift estimates. The best we can do to estimate the cluster membership of a galaxy is to compute the probability for the given galaxy to be part of the cluster. We describe the two possible cases below.

(1) The galaxy only has a photometric redshift. We chose here to consider full probability distribution functions from the official CFHTLS T0007 data release ${ }^{3}$;

(2) The galaxy has a spectroscopic redshift. In this case, we only kept the spectroscopic redshift even when a photometric redshift was available. We assumed for the spectroscopic redshifts a Gaussian presence PDF with a full width at halfmaximum (FWHM) of $150 \mathrm{~km} \mathrm{~s}^{-1}$, typical of the redshift uncertainties of spectroscopic surveys within the CFHTLS W1 (see Adami et al. 2018).

Knowing the position on the sky, magnitude in the $r$-band, error on the magnitude and photometric redshift PDF for a galaxy, as well as the cluster position on the sky and the

see http://cesam.lam. fr/cfhtls-zphots/index/download 
photometric redshift PDF, we implemented the method presented in Castignani \& Benoist (2016; CB16 hereafter) to obtain the probability $P_{\text {mem }}$ for the galaxy gal to belong to a group $G$. We refer to the original paper presenting the method for details (CB16) and provide here a brief outline of the method and of our choice of parameters.

In the framework developed by CB16, the probability membership is obtained using the Bayes theorem,

$$
\begin{aligned}
P_{\text {mem }} \equiv & P\left(\text { gal } \in G \mid \mathcal{P}_{\mathrm{gal}}(z), m_{\mathrm{gal}}^{r},(\mathrm{RA}, \mathrm{Dec})_{\mathrm{gal}},\right. \\
& \left.\times \mathcal{P}_{G}(z), z_{G},(\mathrm{RA}, \mathrm{Dec})_{G}\right) \\
P_{\mathrm{mem}} \propto & P\left(\mathcal{P}_{\mathrm{gal}}(z) \mid \mathrm{gal} \in G\right) P(\mathrm{gal} \in G)
\end{aligned}
$$

where $P\left(\mathcal{P}_{\text {gal }}(z) \mid\right.$ gal $\left.\in G\right)$ is the likelihood of observing the galaxy photometric redshift $\operatorname{PDF} \mathcal{P}_{\text {gal }}(z)$ knowing that the galaxy belongs to the group $G$, and $P($ gal $\in G)$ is the prior probability that the galaxy belongs to the group.

Following CB16, the likelihood is taken to be

$$
P\left(\mathcal{P}_{\text {gal }}(z) \mid \text { gal } \in G\right)=\int \mathcal{P}_{\text {gal }}(z) \mathcal{P}_{G}(z) \mathrm{d} z \text {. }
$$

We have written convolutions as indefinite integrals. We note that in practice, they are discrete sums sampled at the redshift intervals $\mathrm{d} z=0.02$ and taken in the range $0<z<6$ for which the photometric redshift PDF has been calculated.

The prior is computed using the relative number density of group and background galaxies in cylindrical shells around the group. Number densities $n$ are computed as a function of magnitude $m$ and redshift $z$ using the magnitude and photometric redshift PDFs,

$n(m, z)=\frac{1}{A(z)} \sum_{\mathrm{gal}} \mathcal{P}_{\mathrm{gal}}(z) \mathcal{P}_{\mathrm{gal}}(m)$,

where $A(z)$ is the area of the shell in $\operatorname{deg}^{2}$ at redshift $z$ and the magnitude and photometric redshift PDFs are sampled in bins of magnitude $d m=0.1$ and redshift $d z=0.02$.

To mitigate errors due to low number counts in small volumes, we proceeded as in CB16 and used means within a running window of $\pm 5 \mathrm{dm}$ and $\pm \sigma_{z, 95}(\mathrm{~m}, z)$, which is the median $95 \%$ confidence limit on individual photo- $z$ in the CFHTLS in bins of magnitude and redshift. We refer to CB16 for more details. Following their formalism and notations, the prior probability for a galaxy gal of magnitude $m_{\text {gal }}^{r}$ at a projected distance $r_{\text {gal- } G}$ from the group center to be a member of group $G$ located at $z_{G}$ is taken to be

$P($ gal $\in G)=1-\frac{\left\langle n_{\mathrm{bkg}}^{\text {loc }}\left(m_{\mathrm{gal}}^{r}, z_{G}\right)\right\rangle}{\left\langle n_{\mathrm{tot}}\left(m_{\mathrm{gal}}^{r}, z_{G}, r_{\mathrm{gal}-G}\right)\right\rangle}$,

where $n_{\mathrm{bkg}}^{\text {loc }}$ is the background number density computed in an annulus between 3 and $5 \mathrm{Mpc}$ and $n_{\text {tot }}$ is the number density in the considered shell.

The total number density as a function of distance to the cluster was averaged in annuli (shells) around the cluster center offset from each other by $50 \mathrm{kpc}$ and with area of a disk of radius $r=350 \mathrm{kpc}$ at the cluster redshift. This value differs from that of CB16 $(r=450 \mathrm{kpc})$. We chose this because the lowestmass groups in our sample have $R_{200} \sim 350 \mathrm{kpc}$. This average on shells has the advantage of minimizing the centering error on AMASCFI clusters.

The final estimate of the probability membership was obtained by applying a rescaling inspired by that of CB16, that is, normalizing by the maximum probability that would be reached if the galaxy photometric redshift $\operatorname{PDF} \mathcal{P}_{\text {gal }}(z)$ were centered at the group redshift $z_{G}$,

$P_{\text {mem }}=\frac{P\left(\mathcal{P}_{\text {gal }}(z) \mid \text { gal } \in G\right) P(\text { gal } \in G)}{P\left(\mathcal{P}_{\text {gal }}\left(z-z_{G}\right) \mid \text { gal } \in G\right)}$.

\subsection{Estimating the probability for a structure to be a fossil group}

The canonical definition of a FG can be found in Jones et al. (2003). This includes a two-magnitude gap between the BGG and the second brightest galaxy within half a Virial radius, and conditions on the X-ray luminosity of the structure. We here consider the magnitude criterion within half a Virial radius. We therefore computed the probability for a given structure of galaxies to be a FG. This was done knowing the previous membership probabilities and computing the probability of the BGG to be two magnitudes brighter than the second brightest galaxy. This calculation is complicated by the fact that each galaxy has a certain probability of belonging to the group. We define the following events:

$-F$ : the group is fossil,

- $Y_{i}$ : galaxy $i$ is brighter than all other group galaxies by at least two magnitudes,

- $Z_{i}$ : galaxy $i$ belongs to the group,

- $m_{i j}(2)$ : galaxy $j$ has a magnitude brighter than that of galaxy $i$ by two magnitudes,

and their associated probabilities, $P(F), P\left(Y_{i}\right), P\left(Z_{i}\right)$ (computed in the previous subsection), and $P\left(m_{i j}(2)\right)$. The last probability is easily computed by comparing the magnitudes of galaxies $i$ and $j$,

$P\left(m_{i j}(2)\right)=\left\{\begin{array}{ll}0 & \text { if } m_{i}+2<m_{j} \\ 1 & \text { if } m_{i}+2>m_{j}\end{array}\right.$.

The probability $P\left(Y_{i}\right)$ corresponds to the probability that galaxy $i$ belongs to the group and that every galaxy $j$ satisfying $m_{i}+2>m_{j}$ does not belong to the same group,

$P\left(Y_{i}\right)=P\left(Z_{i}\right) \times \prod_{j}\left(1-P\left(Z_{j}\right) P\left(m_{i j}(2)\right)\right)$.

We note that a galaxy $i$ verifying $Y_{i}$ is also the BGG because the magnitude criterion of the FG definition is more restrictive than that of being the BGG (by two magnitudes).

The probability of the group to be fossil is the probability that at least one of the galaxies $i$ is brighter than all other group galaxies by at least two magnitudes, or equivalently, that at least one of the $Y_{i}$ is true,

$P(F)=\sum_{i} P\left(Y_{i}\right)$

\subsection{Is our estimated spatial density of fossil groups realistic?}

Jones et al. (2003) found about 3 to $8 \times 10^{-7}$ fossil groups per $\mathrm{Mpc}^{3}$ (with $h_{70}$ ) for the most massive groups. The precision on the FG density in our analysis is affected by several caveats. The mass estimates of Sarron et al. (2018) come from a scaling relation between richness and X-ray luminosity and therefore have a significant uncertainty that is due to the calibration. This caveat is reasonable, however, because we only applied a cut in mass to select the fossil group sample according to Jones et al. (2003). Second, given our probabilistic approach, we cannot be certain 


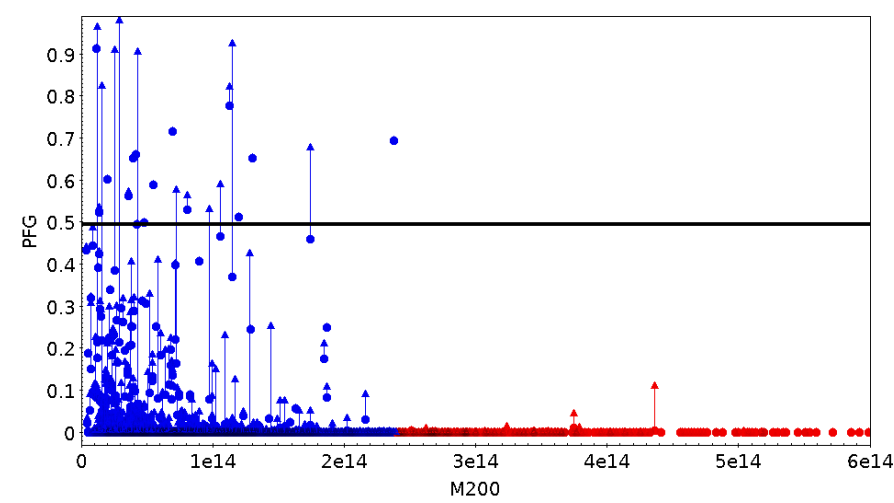

Fig. 1. PFG probability for the structures to be FGs as a function of their estimated $M_{200}$ (Sarron et al. 2018). The vertical blue arrows show the PFG increase when a 20\% ICL additional contribution to the BCG (or BGG) magnitude is allowed. Blue dots are galaxy structures less massive than $2.4 \times 10^{14} M_{\odot}$, and red dots are more massive than $2.4 \times 10^{14} M_{\odot}$. The horizontal black line shows the $50 \%$ level for PFG.

Table 1. Number of structures for different levels of probability to be a fossil group, assuming or not assuming a $20 \%$ contribution by the dominant galaxy.

\begin{tabular}{lcc}
\hline \hline BCG/BGG contribution & None & $20 \%$ \\
\hline PFG $\geq 50 \%$ & 15 & 25 \\
PFG $\geq 15 \%$ & 62 & 85 \\
PFG $\leq 10 \%$ & 2535 & 2510 \\
\hline
\end{tabular}

that a given FG candidate is actually a fossil group. The average sample of high-probability FGs should nonetheless be representative of the true FG sample.

We therefore made an attempt to compute the spatial density of our FG. Linearly extrapolating the Fig. 1 of Sarron et al. (2018) down to low masses, we should have a $\leq 60 \%$ detection rate for typical fossil groups (and a purity $\leq 80 \%$ ). When we sum all the percentages of being a fossil group that we computed for all the structures within the catalog of Sarron et al. (2018), this gives the statistical number of real FGs in this catalog. This computation gives 28.9 FGs within the catalog. Assuming a $\leq 60 \%$ detection rate and a purity of $\leq 80 \%$, we therefore predict about 38.6 fossil groups within the CFHTLS Wide survey. The CFHTLS Wide sampled volume is about $2.43 \times 10^{8} \mathrm{Mpc}^{3}$, leading to a density of $\sim 1.6 \times 10^{-7} \mathrm{FGs}$ per $\mathrm{Mpc}^{3}$. This is of the same order as the estimates from Jones et al. (2003).

\subsection{Caveats}

A possible source of uncertainties in our study is related to the structure of the Brightest Cluster Galaxies (BCG hereafter) themselves. These peculiar galaxies often have very weak and extended halos that are very difficult to detect and separate from the intrastructure diffuse light. When classical flux measurement tools such as Sextractor are applied to the CFHTLS data, part of this contribution might sometimes be missed and is not included in the galaxy CFHTLS magnitude measurements. We may therefore underestimate the luminosity of the BCGs. This strongly depends on the considered BCG (or BGG) and on the history of its parent structure, but Martizzi et al. (2014), for instance, estimated the BCG-related Intra Cluster Light (ICL hereafter) contribution to values higher than $20 \%$ of the total BCG

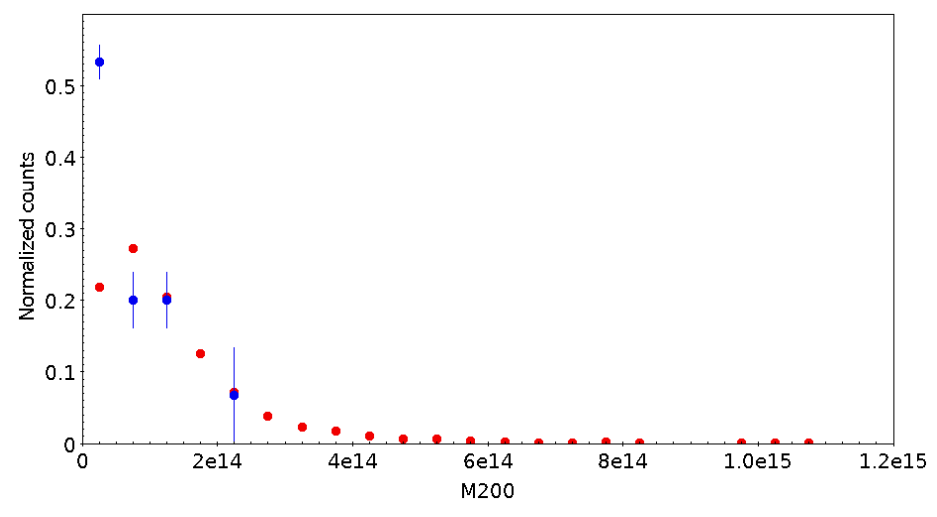

Fig. 2. Arbitrarily normalized counts of the estimated $M_{200}$ masses (in $M_{\odot}$ units) of the AMASCFI candidate structures. Red dots represent the whole sample, and blue dots show structures with a probability higher than $50 \%$ to be a FG. Both samples are normalized. Error bars are Poissonian.

luminosity. All this contribution is not missed when magnitudes are measured in the CFHTLS survey, but even when only $20 \%$ are missed, this may disfavor the FG probability estimate because the value of the BCG magnitude is overestimated. In order to test this effect, we reran our codes assuming this $20 \%$ missing luminosity. This resulted in diminishing the considered two-magnitude gap by $\sim 0.2$ mag within the PFG computation.

This $20 \%$ contribution will increase the number of potential FGs, reaching 25 structures with a PFG value higher than $50 \%$ (15 structures without the $20 \%$ increase). However, this does not change the fact that such structures have masses preferentially lower than $2.4 \times 10^{14} M_{\odot}$ (see Fig. 1).

As a summary, we present in Table 1 the different numbers of structures for different levels of probability to be a FG. We also list the numbers when a $20 \%$ contribution by the dominant galaxy is assumed.

\section{General structure properties versus PFG value}

\subsection{PFG versus $M_{200}$}

We test in this subsection the general behavior of the structures detected in the CFHTLS Wide survey (Sarron et al. 2018) in terms of variation of their $M_{200}$ as a function of their probability PFG to be a fossil group. Figure 1 shows that the more massive a structure, the less likely it is to be a FG, in the sense that the PFG versus $M_{200}$ space is basically empty above $M_{200} \sim$ $2.4 \times 10^{14} M_{\odot}$. Adding a $20 \%$ ICL contribution to the BCG or BGG magnitude does not change this separation strongly. When we limit our analysis to structures with PFG $\geq 50 \%$, we also see that these are low-mass structures (see Fig. 2), mainly lower than $1.5 \times 10^{14} M_{\odot}$.

This value of $50 \%$ is a good compromise between the highest possible probability to be a FG and the sample size. We detected 15 structures with such a probability, which are described in Table A.1.

\subsection{Spatial distribution relative to the nodes and filaments}

One of the key questions when fossil groups are studied is to know where they reside relative to the cosmic web. They might be located in completely isolated regions, as suggested, for example, by Adami et al. (2012), or they might have a distribution similar to that of other galaxy structures. The answer 


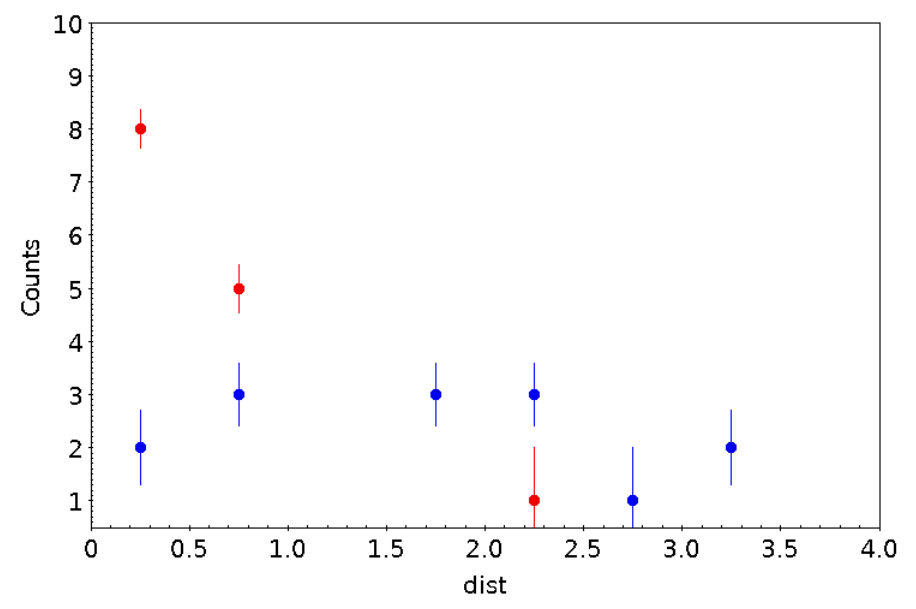

Fig. 3. Distributions of comoving distances (in Mpc) to the nearest filament (red dots) or node (blue dots) of the candidate FGs with $\mathrm{PFG} \geq 50 \%$.

to these questions is a key driver of the building mechanisms of these peculiar structures. The question is how the large magnitude gap between the first and second brightest galaxies can be explained. They might just lack infalling galaxies, which would mean that merging events dominate infalling events. Alternatively, these infalling galaxies might have peculiar impact parameters, which would complicate a possible capture by the structure.

Figure 3 shows that the structures with the PFG values higher than $50 \%$ are preferentially closer to the filaments than to the nodes. While their distribution relative to the nodes is more or less uniform, they exhibit a clear tendency to be located closer than $1 \mathrm{Mpc}$ to their nearest filament. More precisely, $87 \%$ are closer than $1 \mathrm{Mpc}$ to the filaments, while $67 \%$ of them are farther away than $1 \mathrm{Mpc}$ from the nodes. Moreover, we recall that the filament detection completeness is about $70 \%$, which means that structures with PFG values higher than $50 \%$ may all be close to filaments.

We are aware that our statistics are weak (only 15 structures with $\mathrm{PFG} \geq 50 \%$ ), in good agreement with the paucity of this class of objects. Being $\geq 10$ times more extended than the CFHTLS, the Kilo-Degree (KiDS) survey (de Jong et al. 2015) and later the EUropean Cosmic aLl sky Investigator of the Dark universe (EUCLID) surveys (Laureijs et al. 2011) will be much better adapted to detect large populations of FGs when the filaments and nodes will be detected in these areas.

\subsection{Structure distribution within the cosmic web as a function of PFG and mass}

The previous part seems to show that structures with a PFG value higher than $50 \%$ preferentially reside close to the filaments and relatively far from the cosmic web nodes. We investigated whether this is related to the mass of the considered structures. To determine this, we split the whole structure sample into masses higher than $2.4 \times 10^{14} M_{\odot}$, lower than $2.4 \times 10^{14} M_{\odot}$, and lower than $5 \times 10^{13} M_{\odot}$. We also computed the ratio between the distance to the nearest node and to the nearest filament (NtoF hereafter). A high value of NtoF indicates that the considered structure is closer to its nearest filament than to its nearest node. Figure 4 (upper part) shows the general behavior of the NtoF ratio. NtoF is close to 1 for the majority of the structures (as seen in the figure, where the dominant contribution occurs slightly
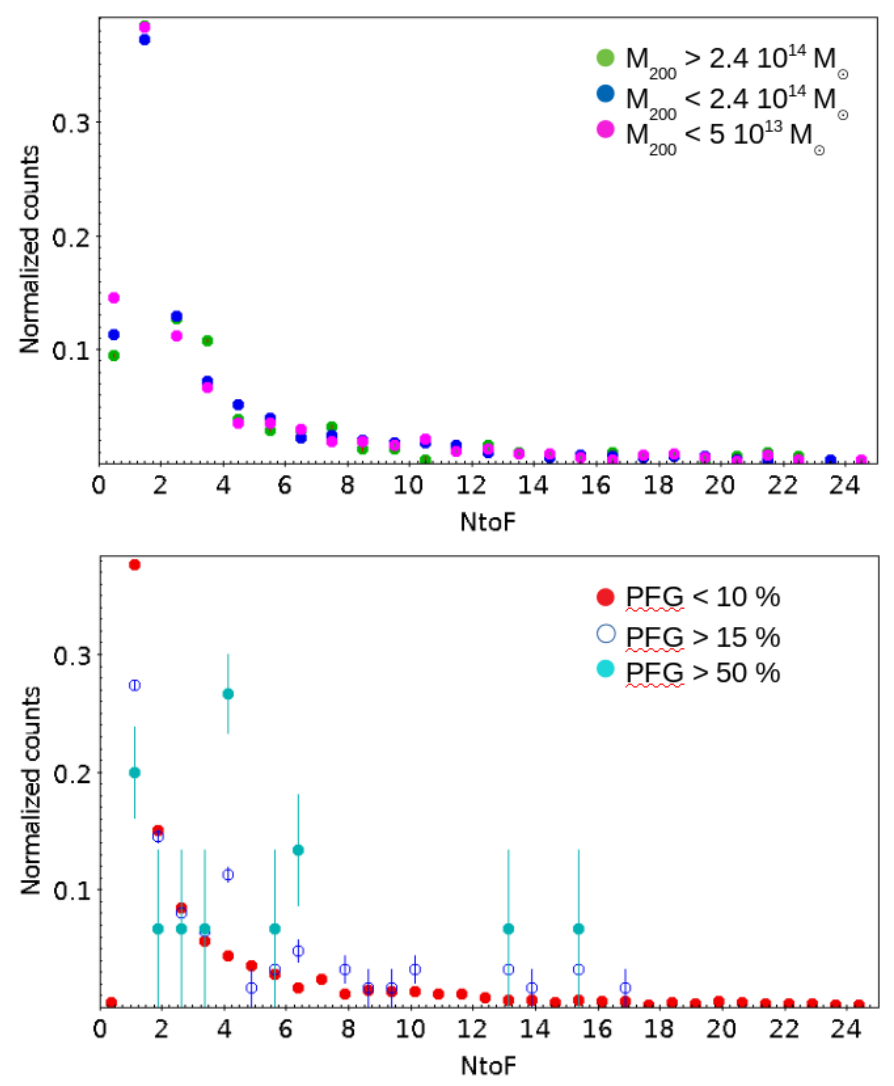

Fig. 4. Upper panel: arbitrarily normalized counts of the ratios NtoF between the distance to the nearest node and to the nearest filament for different structure masses. Structures more massive than $2.4 \times 10^{14} M_{\odot}$ are shown in green, and structures less massive than $2.4 \times 10^{14} M_{\odot}$ are plotted in blue. Structures less massive than $5 \times 10^{13} M_{\odot}$ are shown in magenta. Lower panel: arbitrarily normalized counts of the ratios NtoF between the distance to the nearest node and to the nearest filament for different PFG values. A PFG lower than $10 \%$ is plotted with filled red symbols, a PFG higher than $15 \%$ with open blue symbols, and a PFG higher than $50 \%$ with filled cyan symbols. Error bars are Poissonian and smaller than the point size in the upper panel.

above NtoF $\sim 1$ ). Such structures are equally distant from nodes or filaments. The figure also exhibits a tail of structures closer to filaments than to nodes ( $\mathrm{NtoF} \geq 1)$. This is an expected behavior because filaments cover a larger cosmic volume than nodes. We see no significant effect as a function of mass: a KolmogorovSmirnov test shows that the probability is lower than $0.1 \%$ for the three distributions to differ.

Figure 4 (lower part) is similar, but focuses on the PFG value effect rather than on the mass effect. The structures that are the most different from the FG status (PFG lower than 10\%) have a similar behavior as in Fig. 4 (upper part), with a peak around a $\mathrm{NtoF} \sim 1$. This means that they are not significantly more distant from the nodes than from the filaments. Intermediate structures (PFG higher than 15\%) may be more distant from the nodes, with a larger number of them exhibiting higher NtoF values. The difference between structures with PFG lower than $10 \%$ and PFG higher than $15 \%$ is not very significant, however: a Kolmogorov-Smirnov test predicts a probability of only $7 \%$ for the two distributions to be different. For the structures with the highest PFG value (higher than 50\%), the situation is different. These structures exhibit a preferential NtoF value of $\sim 4$, which is significantly different from the abundances of the other samples at the same NtoF value. They are statistically about four 
times less distant from a filament than from a node. Another peak is present around $\mathrm{NtoF} \sim 6.5$, but is only barely significant because of its large error bar. A Kolmogorov-Smirnov test predicts a probability of $93 \%$ for the distribution with PFG values higher than $50 \%$ to be different from the distribution with PFG values lower than $10 \%$. To summarize, the higher the probability of being a FG, the closer these structures appear to be to the filaments.

\section{Conclusion}

The two main conclusions of this study have been reached by probabilistic arguments and can be summarized as follows:

(1) Only the less massive of our candidate clusters $\left(M_{200} \leq\right.$ $\left.2.4 \times 10^{14} M_{\odot}\right)$ can have a high probability of being FGs.

(2) Structures with the highest probabilities of being FGs are preferentially close to their nearest cosmic filament $(\leq 1 \mathrm{Mpc})$, and their NtoF ratio is preferentially of $\sim 4$.

It is therefore tempting to say that fossil groups reside in cosmological filaments but do not survive in cosmic nodes. This can be explained because in the nodes, the merging rate of structures and/or the galaxy infalling rate are too high and are not compensated for by the intragroup galaxy-galaxy merging rate to maintain the two-magnitude gap observed in FGs. In the filaments, the density (both in terms of field galaxies and other structures) is lower than in the nodes. Fossil groups can therefore empty their vicinity, and their mass evolution is eventually stopped by a lack of accretable material. Another explanation would be that the relative velocities of the group-infalling galaxies are high enough in the filaments to prevent galaxies from being captured by such low-mass galaxy structures. In both cases, the galaxygalaxy intragroup mergers depopulate the group and create the two-magnitude gap in the group galaxy population.

We are aware that these conclusions are based on probabilistic arguments. Only intensive spectroscopic surveys will allow placing these results on firmer grounds.

Acknowledgements. The authors thank the referee. The authors thank Clotilde Laigle for useful discussions. We gratefully acknowledge financial support from the Centre National d'Etudes Spatiales (CNES) for many years. NM acknowledges support by a CNES fellowship. Based on observations obtained with $X M M-N e w t o n$, an ESA science mission with instruments and contribution directly funded by ESA Member States and NASA. Based on observations made with ESO Telescopes at the La Silla and Paranal Observatories under programmes ID 191.A-0268 and 60.A-9302. Based on observations obtained with MegaPrime/MegaCam, a joint project of CFHT and CEA/IRFU, at the CanadaFrance-Hawaii Telescope (CFHT) which is operated by the National Research Council (NRC) of Canada, the Institut National des Sciences de l'Univers of the Centre National de la Recherche Scientifique (CNRS) of France, and the University of Hawaii. This work is based in part on data products produced at Terapix available at the Canadian Astronomy Data Centre as part of the CanadaFrance-Hawaii Telescope Legacy Survey, a collaborative project of NRC and CNRS. This research has made use of the VizieR catalogue access tool, CDS Strasbourg, France. This research has also made use of the NASA/IPAC Extragalactic Database (NED) which is operated by the Jet Propulsion Laboratory, California Institute of Technology, under contract with the National Aeronautics and Space Administration. This research has made use of the ASPIC database, operated at CeSAM/LAM, Marseille, France. This paper uses data from the VIMOS Public Extragalactic Redshift Survey (VIPERS). VIPERS has been performed using the ESO Very Large Telescope, under the "Large Programme" 182.A-0886. The participating institutions and funding agencies are listed at http://vipers.inaf.it. Based on data obtained with the European Southern Observatory Very Large Telescope, Paranal, Chile, under Large Program 185.A-0791, and made available by the VUDS team at the CESAM data center, Laboratoire d'Astrophysique de Marseille, France. This research uses data from the VIMOS VLT Deep Survey, obtained from the VVDS database operated by Cesam, Laboratoire d'Astrophysique de Marseille, France. GAMA is a joint European-Australasian project based around a spectroscopic campaign using the Anglo-Australian Telescope. The GAMA input catalogue is based on data taken from the Sloan Digital Sky Survey and the UKIRT Infrared Deep Sky Survey. Complementary imaging of the GAMA regions is being obtained by a number of independent survey programmes including GALEX MIS, VST KiDS, VISTA VIKING, WISE, Herschel-ATLAS, GMRT and ASKAP providing UV to radio coverage. GAMA is funded by the STFC (UK), the ARC (Australia), the AAO, and the participating institutions. The GAMA website is http: //www. gama-survey.org/. This paper uses data from the XXL survey, an international project based around an XMM Very Large Programme surveying two $25 \mathrm{deg}^{2}$ extragalactic fields at a depth of $6 \times 10^{-15} \mathrm{erg} \mathrm{cm}^{2} \mathrm{~s}^{-1}$ in the $[0.5-2] \mathrm{keV}$ band for point-like sources. The XXL website is http: //irfu.cea.fr/xxl. Multi-band information and spectroscopic follow-up of the X-ray sources are obtained through a number of survey programmes, summarised at http://xxlmultiwave.pbworks.com/.

\section{References}

Adami, C., \& Mazure, A. 1999, A\&AS, 134, 393

Adami, C., Jouvel, S., Guennou, L., et al. 2012, A\&A, 540, A105

Adami, C., Giles, P., Koulouridis, E., et al. 2018, A\&A, 620, A5

Arnouts, S., Cristiani, S., Moscardini, L., et al. 1999, MNRAS, 310, 540

Baldry, I. K., Liske, J., Brown, M. J. I., et al. 2018, MNRAS, 474, 3875

Bertin, E., \& Arnouts, S. 1996, A\&AS, 117, 393

Bharadwaj, V., Reiprich, T. H., Sanders, J. S., \& Schellenberger, G. 2016, A\&A, 585, A 125

Castignani, G., \& Benoist, C. 2016, A\&A, 595, A111

Coleman, G. D., Wu, C. C., \& Weedman, D. W. 1980, ApJS, 43, 393

Coupon, J., Ilbert, O., Kilbinger, M., et al. 2009, A\&A, 500, 981

de Jong, J. T. A., Verdoes Kleijn, G. A., Boxhoorn, D. R., et al. 2015, A\&A, 582, A62

D’Onghia, E., Sommer-Larsen, J., Romeo, A. D., et al. 2005, ApJ, 630, L109

Girardi, M., Aguerri, J. A. L., De Grandi, S., et al. 2014, A\&A, 565, A115

Gozaliasl, G., Finoguenov, A., Khosroshahi, H. G., et al. 2014, A\&A, 566, A140

Guennou, L., Adami, C., Ulmer, M. P., et al. 2010, A\&A, 523, A21

Ilbert, O., Arnouts, S., McCracken, H. J., et al. 2006, A\&A, 457, 841

Jones, L. R., Ponman, T. J., Horton, A., et al. 2003, MNRAS, 343, 627

Jones, D. H., Read, M. A., Saunders, W., et al. 2009, MNRAS, 399, 683

Kim, D.-W., Anderson, C., Burke, D., et al. 2018, ApJ, 853, 129

Kinney, A. L., Calzetti, D., Bohlin, R. C., et al. 1996, ApJ, 467, 38

La Barbera, F., de Carvalho, R. R., de la Rosa, I. G., et al. 2009, AJ, 137, 3942

Laigle, C., Pichon, C., Arnouts, S., et al. 2018, MNRAS, 474, 5437

Laureijs, R., Amiaux, J., Arduini, S., et al. 2011, ArXiv e-prints [arXiv:1110.3193]

Le Fèvre, O., Vettolani, G., Garilli, B., et al. 2005, A\&A, 439, 845

Le Fèvre, O., Cassata, P., Cucciati, O., et al. 2013, A\&A, 559, A14

Lilly, S. J., Le Brun, V., Maier, C., et al. 2009, ApJS, 184, 218

Lima Neto, G. B., Durret, F., Laganá, T. F., et al. 2020, A\&A, in press, https: //doi.org/10.1051/0004-6361/201936098

Martinet, N., Durret, F., Guennou, L., et al. 2015, A\&A, 575, A116 Martizzi, D., Jimmy, Teyssier, R., \& Moore, B. 2014, MNRAS, 443, 1500

Mirkazemi, M., Finoguenov, A., Pereira, M. J., et al. 2015, ApJ, 799, 60

Ponman, T. J., Allan, D. J., Jones, L. R., et al. 1994, Nature, 369, 462

Santos, W. A., de Oliveira, C. M., \& Sodré Jr., L. 2007, AJ, 134, 1551

Sarron, F., Martinet, N., Durret, F., \& Adami, C. 2018, A\&A, 613, A67

Sarron, F., Adami, C., Durret, F., \& Laigle, C. 2019, A\&A, 632, A49

Scodeggio, M., Guzzo, L., Garilli, B., et al. 2018, A\&A, 609, A84

Sousbie, T. 2011, MNRAS, 414, 350

Tasca, L. A. M., Le Fèvre, O., Ribeiro, B., et al. 2017, A\&A, 600, A110

von Benda-Beckmann, A. M., D’Onghia, E., Gottlöber, S., et al. 2008, MNRAS, 386,2345

Zarattini, S., Girardi, M., Aguerri, J. A. L., et al. 2016, A\&A, 586, A63 
Appendix A: List of the 15 galaxy structures with PFG $\geq 50 \%$

Table A.1. 15 structures from Sarron et al. (2018) with PFG $\geq 50 \%$.

\begin{tabular}{|c|c|c|c|c|c|c|c|c|c|c|c|}
\hline CFHTLS field & $\begin{array}{c}\text { RA } \\
\text { (deg) } \\
\end{array}$ & $\begin{array}{c}\text { RA uncertainty } \\
\text { (deg) }\end{array}$ & $\begin{array}{c}\text { Dec } \\
(\operatorname{deg})\end{array}$ & $\begin{array}{c}\text { Dec uncertainty } \\
(\mathrm{deg})\end{array}$ & $S / N$ & Redshift & $\begin{array}{c}M_{200} \\
M_{\odot} \\
\end{array}$ & $\begin{array}{l}R_{200} \\
\mathrm{Mpc} \\
\end{array}$ & $\begin{array}{c}\text { dnode } \\
\text { Mpc }\end{array}$ & $\begin{array}{c}\text { dfil } \\
\mathrm{Mpc}\end{array}$ & $\begin{array}{c}\text { PFG } \\
\% \\
\end{array}$ \\
\hline W1 & 34.72 & 0.05 & -9.27 & 0.05 & 6 & 0.22 & $4.20 \mathrm{E} 13$ & 0.78 & 1.77 & 0.51 & 50 \\
\hline W1 & 36.76 & 0.03 & -9.27 & 0.03 & 4 & 0.32 & $1.20 \mathrm{E} 14$ & 1.15 & 3.42 & 0.64 & 51 \\
\hline W1 & 35.89 & 0.05 & -8.69 & 0.05 & 6 & 0.26 & $1.39 \mathrm{E} 13$ & 0.56 & 2.59 & 0.17 & 52 \\
\hline W1 & 32.20 & 0.05 & -6.60 & 0.05 & 3 & 0.20 & $3.97 \mathrm{E} 13$ & 0.77 & 2.10 & 0.50 & 65 \\
\hline W1 & 30.80 & 0.01 & -9.80 & 0.01 & 4 & 0.66 & $1.30 \mathrm{E} 14$ & 1.35 & 0.83 & 0.19 & 65 \\
\hline W1 & 36.82 & 0.02 & -4.55 & 0.02 & 10 & 0.28 & 2.38E14 & 1.47 & 0.81 & 0.21 & 69 \\
\hline W1 & 38.18 & 0.03 & -9.77 & 0.03 & 3 & 0.36 & $6.92 \mathrm{E} 13$ & 1.00 & 2.07 & 0.32 & 71 \\
\hline W1 & 38.69 & 0.03 & -5.64 & 0.03 & 6 & 0.30 & $1.13 \mathrm{E} 14$ & 1.15 & 1.69 & 0.26 & 78 \\
\hline W1 & 37.14 & 0.03 & -5.84 & 0.04 & 4 & 0.30 & $1.15 \mathrm{E} 13$ & 0.55 & 0.51 & 0.57 & 91 \\
\hline W2 & 136.19 & 0.02 & -4.65 & 0.02 & 6 & 0.68 & $8.06 \mathrm{E} 13$ & 1.17 & 0.39 & 0.10 & 53 \\
\hline W2 & 135.46 & 0.04 & -4.33 & 0.04 & 4 & 0.22 & $1.97 \mathrm{E} 13$ & 0.61 & 2.41 & 0.19 & 60 \\
\hline W3 & 213.46 & 0.01 & 54.78 & 0.01 & 6 & 0.56 & $4.75 \mathrm{E} 13$ & 0.94 & 1.92 & 0.80 & 50 \\
\hline W3 & 212.57 & 0.06 & 57.69 & 0.03 & 6 & 0.36 & $3.63 \mathrm{E} 13$ & 0.77 & 3.15 & 2.31 & 56 \\
\hline W3 & 219.40 & 0.07 & 56.55 & 0.04 & 5 & 0.26 & $4.18 \mathrm{E} 13$ & 0.79 & 35.54 & 35.64 & 66 \\
\hline W4 & 334.64 & 0.02 & -0.76 & 0.02 & 7 & 0.36 & $5.48 \mathrm{E} 13$ & 0.93 & 0.16 & 0.09 & 59 \\
\hline
\end{tabular}

Notes. The table lists: (1) the corresponding CFHTLS field, (2-3): RA and uncertainty, (4-5): Dec and uncertainty, (6) detection signal-to-noise ratio (S/N) within the CFHTLS structure sample, (7) redshift, (8) $M_{200},(9) R_{200}$, (10) distance to the nearest node, (11) distance to the nearest filament, (12) probability of being a FG. The structure at coordinates $(219.40,56.55)$ is probably in a region where filament detection has been inoperative. 